\title{
Technique and setup for diagnostics of $p$ - $n$ junction to case thermal resistance in high-power gallium nitride LEDs
}

\author{
V.M. Sorokin ${ }^{1}$, R.V. Konakova', Ya.Ya. Kudryk', A.V. Zinovchuk², \\ R.I. Bigun ${ }^{3}$, R.Ya. Kudryk ${ }^{3}$, V.V. Shynkarenko ${ }^{1}$ \\ ${ }^{1} V$. Lashkaryov Institute of Semiconductor Physics, NAS of Ukraine \\ 41, prospect Nauky, Kyiv 03028, Ukraine \\ Tel.: (380-44) 525-57-85; e-mail:vsorokin@isp.kiev.ua \\ ${ }^{2}$ I. Franko Zhytomyr State University \\ ${ }^{3}$ I. Franko Lvivs National University
}

\begin{abstract}
We present a setup and procedure of studying $p$ - $n$ junction to case thermal resistance in high-power light-emitting diodes (LEDs) from their thermal relaxation. A set of LEDs mounted on a metal-core printed circuit board (MCPCB) were studied. The contributions to the total thermal resistance from a heavy heat sink, MCPCB, heat slug and LED chip are separated.
\end{abstract}

Keywords: light-emitting diode (LED), gallium nitride, heat sink, thermal resistance.

Manuscript received 16.02.12; revised version received 16.03.12; accepted for publication 27.03.12; published online 30.05.12.

\section{Introduction}

Development of novel energy-saving light sources is one of the promising lines in present-day world science and technology whose progress is impossible without metrological assurance. Knowledge of the whole set of parameters and characteristics of LED devices enables one to predict their behavior over a long period of time, optimize their design and improve thermal characteristics, increase energy efficiency factor (EEF) and assure manufacturing of competitive LED devices. In this case, the metrological base requires modern highprecision measuring devices certified in accordance with the international standards. The constituent part of metrology of a LED illuminator is metrology of LED itself (as the primary element of illuminator), especially diagnostics of device thermal state at sufficiently high operating currents.

Here we describe a setup for diagnostics of $p$ - $n$ junction to case thermal resistance in high-power LEDs as well as research technique that can give information on thermal characteristics of LED chip and structure parts.
For LED characterization, the equivalent thermal resistance $R_{e f}$ is used most often. It equals to the ratio between the difference of $p-n$ junction and heat sink temperatures $\Delta T$ and total supplied power $P$ :

$$
R_{e f}=\frac{\Delta T}{P}=\frac{\Delta T}{V I}
$$

here $V$ is LED voltage and $I$ is LED current.

On the other hand, for physical calculations involving thermal parameters of materials, one should determine the $p$ - $n$ junction-LED case thermal resistance $R_{t h}$ that takes into account not the total scattered power but its thermal part only [1]:

$$
R_{t h}=\frac{\Delta T}{P_{t h}}=\frac{\Delta T}{P(1-\eta)}=R_{e f} \frac{1}{1-\eta},
$$

where $P_{t h}$ is the thermal power and $\eta$ is LED EEF. However, when describing the dynamics of LED heating or cooling, one should take into account both thermal resistances and thermal capacitances of LED (or system of LEDs) structure parts [1-5]. As thermal power $P_{t h}$ is supplied to $p-n$ junction, its temperature increases 
exponentially rather than abruptly. The heating-up rate depends on material (semiconductor and copper) heat capacity $C_{t h}$ and thermal resistance $R_{t h}$ as well as thermal power supplied. In a case of several regions with different thermal conductivities and heat capacities (i.e., different thermal relaxation constants $\tau_{t h}=R_{t h} C_{t h}$ ), the LED cooling kinetics is described using a multisectional model (Fig. 1c):

$$
\Delta T=\sum_{i=1}^{n} \Delta T_{i} \exp \left(-\frac{t}{\tau_{t h, i}}\right) .
$$

In the case of LED heating, the LED heating kinetics is of the following form:

$$
\Delta T=\sum_{i=1}^{n} \Delta T_{i}\left(1-\exp \left(-\frac{t}{\tau_{t h, i}}\right)\right),
$$

where $\Delta T_{i}$ is the equilibrium temperature drop at the $i$-th section.

In what follows, we shall estimate the number of sections and their characteristic parameters in the thermal model.

\section{Setup and procedure for measurement of $p$ - $n$ junction to case thermal resistance in LEDs}

The measurement of the equivalent thermal resistance is made in the following way [6-9]. At first one should measure the calibration voltage vs temperature dependence, $V(T)$, at a current $I_{F}$, namely, take nonheating (pulse) $I-V$ curves in the LED package temperature range $20-120^{\circ} \mathrm{C}$. The non-heating condition is provided by pulses of period $\tau_{F}<<\tau_{t h r}$ (where $\tau_{t h r}$ is the characteristic time of LED thermal relaxation). Then a heating current $I_{T}=I_{\max }$ is fed to the LED. $I_{\max }$ is the maximal pulse operating current, with a pulse period $\tau_{T} \gg \tau_{t h r}$ and pulse ratio $Q=\tau_{T} /\left(\tau_{T}+\tau_{F}\right)$, to which a constant direct current $I_{F}$ is added. At the moment of current $I_{T}$ switch-off, one should measure the voltage $V_{F}$ from which the $p$ - $n$ junction temperature $T_{F}$ is determined using the calibration curve. The equivalent LED thermal resistance $R_{e f}$ is determined from the difference of $p$ - $n$ junction and case temperatures using Eq. (1).

Measurement of temperature kinetics is performed in similar way, except that calibration is made at a current $I_{F}=I_{T}$ to increase gaging speed. Then constant heating direct current $I_{T}$ is fed to LED, and LED voltage as function of time is measured. Knowing the temperature, voltage and current at each point of the $I-V$ curve, one can calculate kinetics of the equivalent thermal resistance using Eq. (1).

The block diagram of the setup for investigation of LED thermal resistance LED is presented in Fig. 2. The setup involves a personal computer (PC) with built-in two-channel digital-to-analog and analog-to-digital converters (DAC and ADC, respectively), an external unit (EU) with a voltage-current $(V / I)$ converter and buffer voltage amplifier (2), and a thermostat (Th). Automatic thermoregulation of thermostat was bounded below by room temperature: $T_{\min }=T_{\text {room }}+5 \mathrm{~K}$. The current range was $0-5 \mathrm{~A}$, with step of measurement $1.25 \mathrm{~mA}$. The voltage range was $0-10 \mathrm{~V}$, with step of measurement $2.5 \mathrm{mV}$. The pulse duration began with $8 \mu \mathrm{s}$, with step of measurement $2 \mu \mathrm{s}$. The accuracy of temperature measurement with temperature-sensitive device was $\pm 0.1 \mathrm{~K}$, while the accuracy of temperature maintenance was $\pm 0.3 \mathrm{~K}$. To increase accuracy of measurements, they were made using separate current and potential conductors.
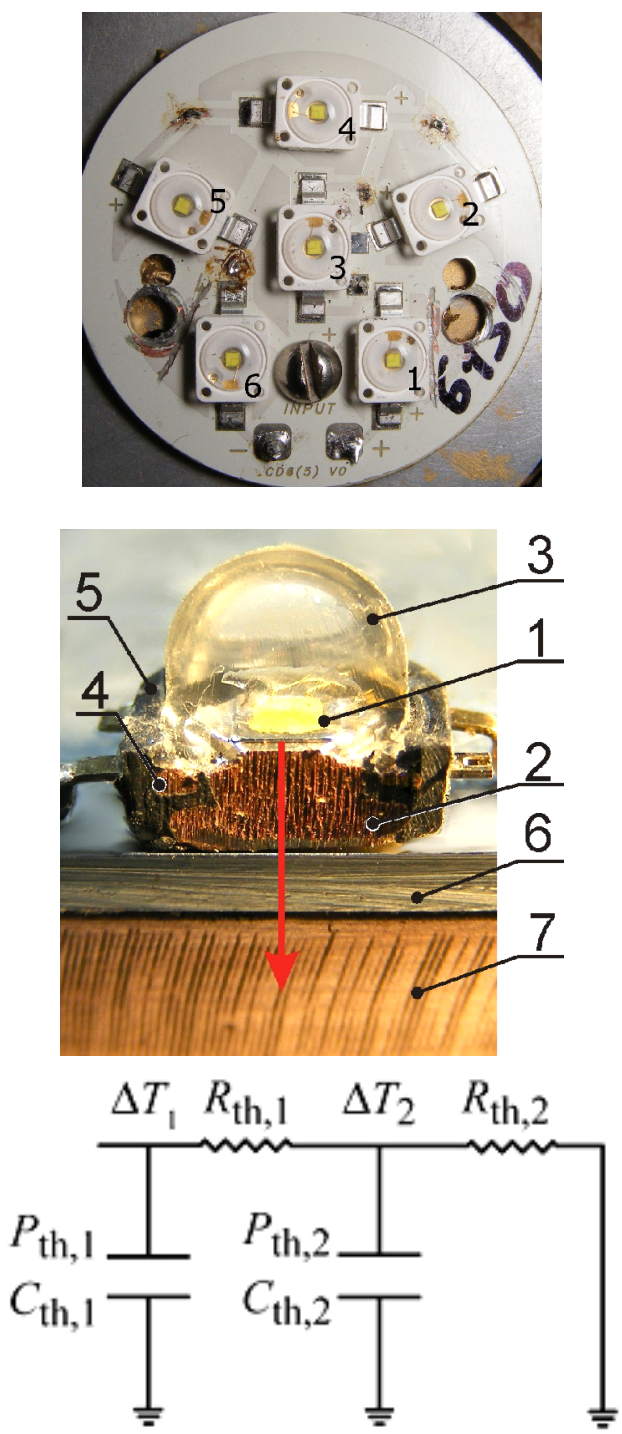

Fig. 1. Appearance of a prototype (a) and schematic of heat flux propagation (b): 1 - LED chip; 2 - copper heat slug; 3 lens; 4 - electrical contact; 5 - plastic case; 6 - Al-based MCPCB; 7 - heavy heat sink. The arrow indicates direction of heat flux propagation. In this design, both electrical leads are connected using microwire welding, so chip-electrical lead thermal resistance is high. (c) LED multi-sectional thermal model. 

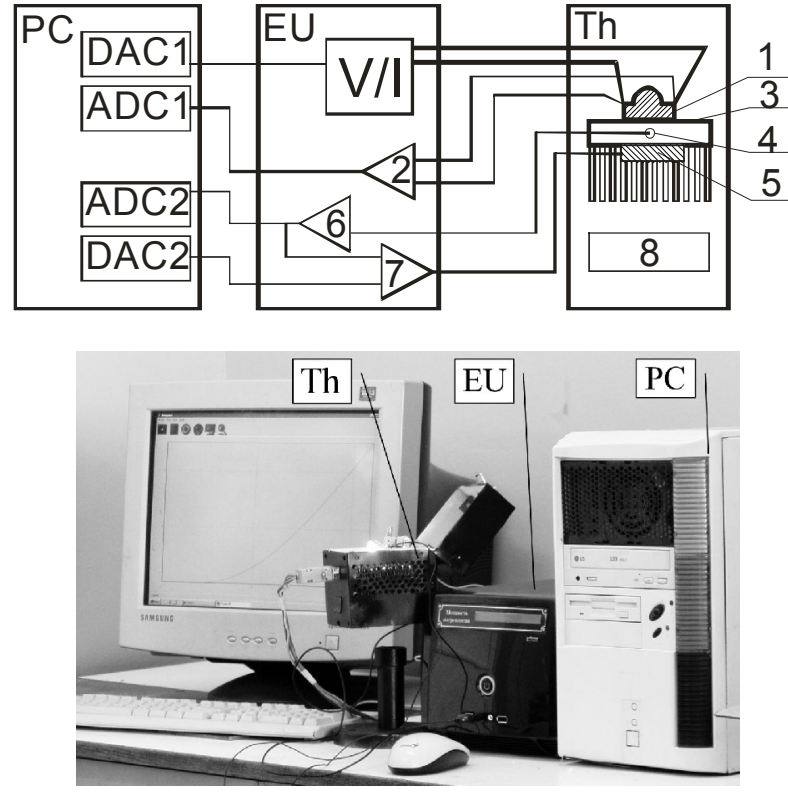

Fig. 2. Block diagram (a) and photograph (b) of the setup for measurement of equivalent thermal resistance: $\mathrm{PC}$ - personal computer, DAC 1 and DAC 2 - the first and second digital-toanalog converter, ADC 1 and ADC 2 - the first and second analog-to-digital converter; EU - external unit; Th thermostat. 1 - specimen, 2 - buffer voltage amplifier; 3 - hot plate; 4 - thermal sensor, 5 - heater, 6 - amplifier of thermal sensor signal, 7 - comparator, 8 - cooler.

\section{Electrophysical properties and features of thermal relaxation in LEDs}

Testing of the setup and measuring technique was performed for a set of gallium nitride LUW_W5AM Golden Dragon plus/OSRAM LEDs [10] on a common heat sink. A set of pulse $I-V$ curves (see Fig. 3) was taken by varying heat sink temperature in the $20-120{ }^{\circ} \mathrm{C}$ range (with step of $10^{\circ} \mathrm{C}$ ) and using short monitoring pulses $(12 \mu \mathrm{s})$ with high pulse ratio $\left(10^{5}\right)$ that did not heat a specimen.

Using the pulse $I-V$ curves, it is possible to build a calibration curve at any current in the $\left(10^{-3}-1\right)$ A range. In particular, Fig. 4 shows calibration curves built at a rated current of $350 \mathrm{~mA}$; one can see a spread of parameters of the LEDs on a common heat sink.

The kinetics of thermal relaxation was determined in the following way. A heating pulse of rated current $(350 \mathrm{~mA})$ was fed to the specimen, and temperature kinetics was taken at the $p$ - $n$ junction, with logarithmic time sampling (Fig. 5). One can see that, at given current and heat sink temperature, pulses with duration of up to $10^{-4} \mathrm{~s}$ did not led to $p-n$ junction heating in the LEDs under investigation, i.e., those pulses were non-heating. Separation of contributions to the temperature relaxation curve from structure parts of the LED module can be done with two techniques, namely, differentiation of the temperature relaxation curve or its approximation using a theoretical model. For comparison we shall make an analysis using both techniques.

In the first case, the temperature relaxation curve is flattened and differentiated. The result obtained is presented in Fig. 6a. One can see that the differentiated curve has five clearly pronounced points that probably correspond to five exponential relationships of a fivesectional model used to describe the relaxation curve. The point of inflection 1 can be identified immediately after detachment of the heavy heat sink. One can see that the heavy heat sink affects the relaxation curve at relaxation times over 2-3 s. And the maximum 2 near $0.4 \mathrm{~s}$ has to correspond to MCPCB at which the LEDs are mounted (Fig. 1b). The peak 3, by analogy with [6], corresponds to the heat slug. The points 5 and 6 (with characteristic time of $5 \times 10^{-4} \mathrm{~s}$ and $10^{-3} \mathrm{~s}$ ) probably correspond to thermal characteristics of the chip and chip-thermal distributor thermal interface.

Along with application of the first technique, approximation with least square procedure of the experimental relaxation curve (Eq. (4)) was performed. When approximating the experimental curve with model one and increasing the number of sections in the theoretical model, one can see from Table 1 that the root-mean-square (RMS) error decreases as the number of sections increases up to four. However, addition of the fifth section does not result in considerable reduction of the RMS error: in this case, the relaxation time practically coincides with one of the times for previous sections, so there is no need to introduce the fifth section in the model.

It is evident that the approximation gives a good description for the experimental curve, except for the initial piece of the curve (Fig. 6b). By comparing the EEF values obtained using approximation (Table 2) and from the $d V / d \log (t)$ dependence on $t$, one can conclude that the highest-frequency section related to the LED chip is not described with exponential. This may be related to the effect (at such short times) of charge relaxation processes in the LED, which requires additional investigation.

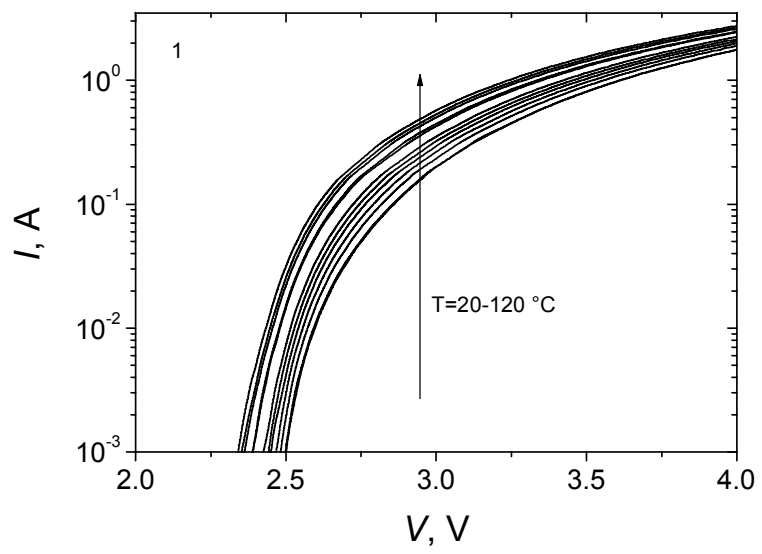

Fig. 3. Pulse $I-V$ curves of a LED mounted on heat sink (Fig. 1a); temperature range $20-120^{\circ} \mathrm{C}$, step of measurement $10^{\circ} \mathrm{C}$. 


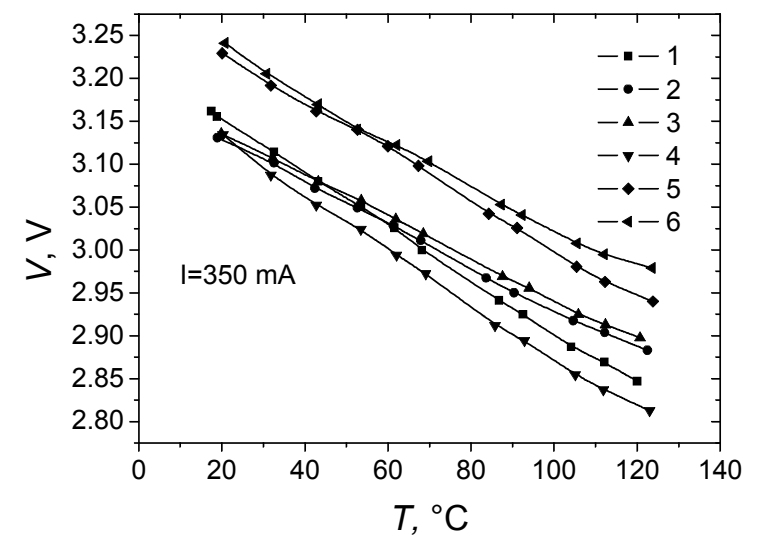

Fig. 4. Dependence of LED voltage on $p$ - $n$ junction temperature at a constant direct current of $350 \mathrm{~mA} \mathrm{(1-6} \mathrm{-} \mathrm{diode}$ number in Fig. 1a).

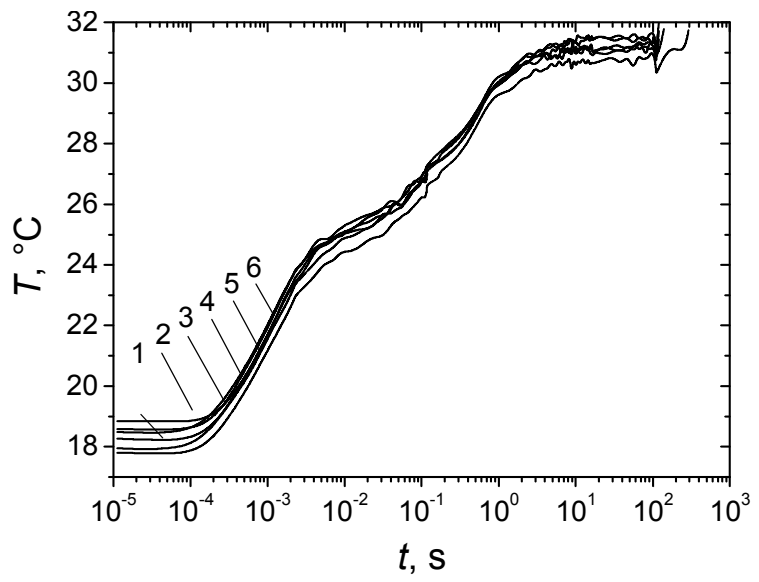

Fig. 5. Temperature relaxation for six LEDs at heating with constant direct current (1-6 - diode number in Fig. 1a).

By assuming that it is possible to neglect heat dissipation through a plastic case and knowing temperature drops $\Delta T_{i}$ at each section, one can calculate the effective thermal resistance for each section in Eq. (1). The results of calculations are in good agreement with the typical parameters for LUW-W5AM LEDs (Table 3): the sums of equivalent thermal resistances of the sections 3 and 4 agree well with the typical values of equivalent thermal resistances for those LEDs. To calculate the $p$ - $n$ junction to case thermal resistance as well as thermal capacitances of each section, we additionally measured EEF at a standardized setup High Accuracy Array Spectrometer Everfine HAAS-2000 (see Table 3).

Table 1. Dependence of minimal RMS approximation error on the number of sections in multi-section model.

\begin{tabular}{|c|c|}
\hline Number of sections & RMS error, \% \\
\hline 2 & 0.038 \\
\hline 3 & 0.022 \\
\hline 4 & 0.019 \\
\hline 5 & 0.019 \\
\hline
\end{tabular}

Table 2. The approximation coefficients for experimental measurements in a four-section model (see dashed curve in Fig. 6b).

\begin{tabular}{|c|c|c|c|c|c|}
\hline $\begin{array}{c}\text { Section } \\
\text { number } \boldsymbol{i}\end{array}$ & $\boldsymbol{\Delta}_{\boldsymbol{i}},{ }^{\circ} \mathbf{C}$ & $\boldsymbol{\tau}_{\boldsymbol{t h r}, \boldsymbol{I}}, \mathbf{S}$ & $\begin{array}{c}\boldsymbol{R}_{\boldsymbol{e f}}, \\
\mathbf{K} / \mathbf{W}\end{array}$ & $\begin{array}{c}\boldsymbol{R}_{\boldsymbol{t} \boldsymbol{h}}, \\
\mathbf{K} / \mathbf{W}\end{array}$ & $\begin{array}{c}\boldsymbol{C}_{\boldsymbol{t h}}, \\
\mathbf{W} \cdot \mathbf{s} / \mathbf{K}\end{array}$ \\
\hline 1 & 1.399 & 2.001 & 1.25 & 1.84 & 1.00 \\
\hline 2 & 3.570 & 0.3884 & 3.19 & 4.69 & 0.0761 \\
\hline 3 & 1.543 & 0.0357 & 1.38 & 2.03 & 0.0162 \\
\hline 4 & 6.049 & $1.31 \times 10^{-3}$ & 5.40 & 7.94 & $1.5 \times 10^{-4}$ \\
\hline
\end{tabular}

Table 3. Typical parameters of LUW W5AM LEDs [10] and check measurements for LED no 1 .

\begin{tabular}{|l|c|c|c|}
\hline \multicolumn{1}{|c|}{ Parameters } & Typical & Maximal & Measured \\
\hline $\begin{array}{l}\text { luminous flux, } \mathrm{lm} \\
\left(\text { at } 350 \mathrm{~mA}, 25^{\circ} \mathrm{C}\right)\end{array}$ & 116 & $112.5^{*}$ \\
\hline $\begin{array}{l}\text { LED voltage, V } \\
\left.\text { (at } 350 \mathrm{~mA}, 25^{\circ} \mathrm{C}\right)\end{array}$ & 3.2 & 3.7 & $3.2^{*}$ \\
\hline $\begin{array}{l}\text { effective thermal } \\
\text { resistance } R_{\text {th }} \text { eff, } \\
\text { K/W }\end{array}$ & 6.5 & 11 & $6.7^{* *}$ \\
\hline $\begin{array}{l}\text { energy efficiency } \\
\text { factor }\end{array}$ & 0.37 & & $0.320^{*}$ \\
\hline
\end{tabular}

*for LED no 1, measuring equipment Everfine HAAS-2000;

${ }^{* *}$ for LED no 1, sum of effective thermal resistances of the third and fourth sections.
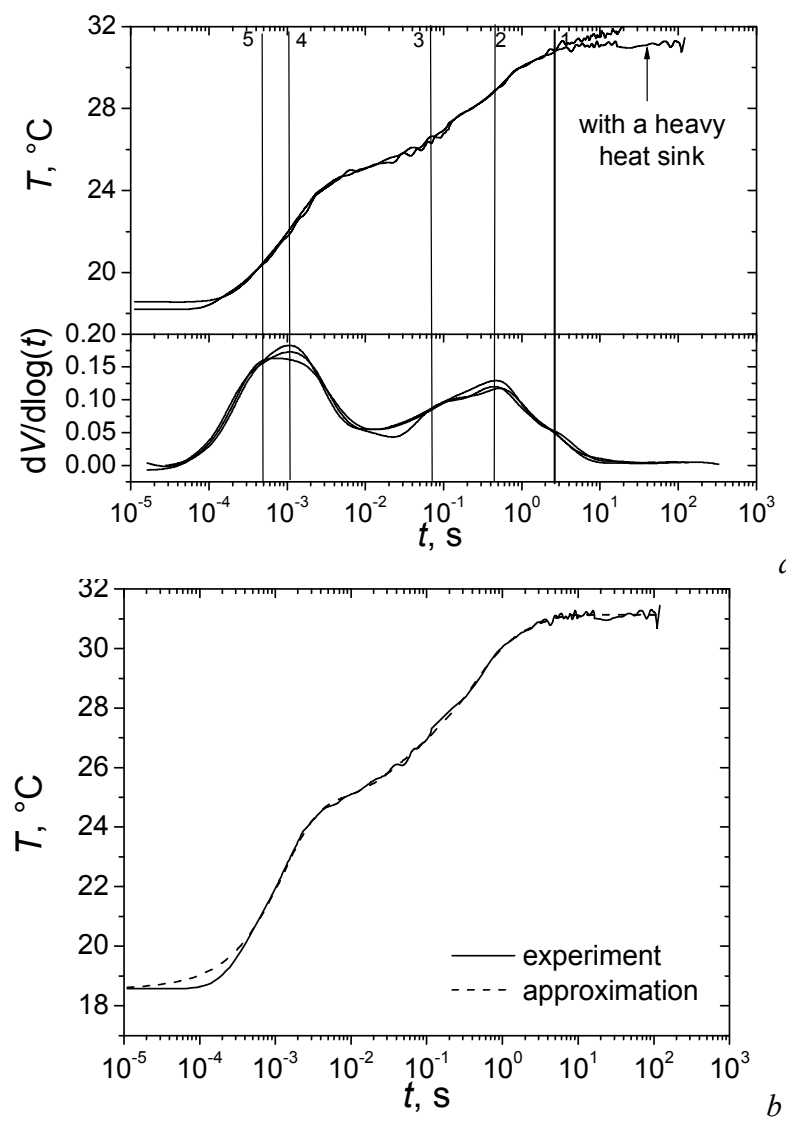

Fig. 6. Time dependence of $d V / d \log (t)$ (a) and four-sectional approximation of LED no 1 temperature relaxation (b). 
The manufacturer did not present the typical LED EEF. However, it can be estimated from other parameters. Knowing the dependence of emission power on wavelength, $J_{e}(\lambda)$, one can determine the total emission power, $J_{W}$, by integrating the above dependence over all possible wavelengths and multiplying the result of integration by normalization coefficient $J_{0}$ :

$J_{W}=J_{0} \int J_{e}(\lambda) d \lambda$.

On the other hand, LED efficiency in lumens, $J_{L m}$, is the integral of the product of spectral emission power $J_{e}(\lambda)$ and visibility curve $\bar{y}(\lambda)$ (with the corresponding coefficient) over all possible wavelengths [11]:

$$
J_{L m}=683.002[L m / W] J_{0} \int \bar{y}(\lambda) J_{e}(\lambda) d \lambda .
$$

After numerical calculation of the integrals in Eqs. (5) and (6), we obtain $J_{L m}=276.8 J_{W}$. So the typical EEF value is equal to the ratio between the emitted power and total dissipated power: $\eta=$ $0.42 /(3.2 \times 0.35)=0.375$.

It should be also noted that, contrary to thermal resistance, the effective thermal resistance additionally depends on temperature owing to the EEF temperature dependence. This has to be taken into account when designing LED sources with high-power LEDs.

\section{Conclusion}

Investigation of thermal relaxation kinetics gives information on thermal resistances, thermal capacitances and thermal relaxation constants of LED structure parts and quality of chip-heat slug and heat slug-printed circuit board thermal interfaces, which can be used at the stage of device development and product test as well.

To build more adequate electrothermal kinetic model for LED, one should take into account the effects related to thermal and electrical nonuniformities in LED, EEF and their variation with LED current and temperature.

\section{Acknowledgements}

This work is part of the project 31/4.2.3.1/1833 of the Government Scientific \& Technical Target Program "Development and introduction of energy-saving LED light sources and illumination systems based on them".

\section{References}

1. F.E. Shubert, Light-Emitting Diodes, Cambridge University Press (2006).

2. Lan Kim, Woong Joon Hwang, Moo Whan Shin, Thermal resistance analysis of high power LEDs with multi-chip package, in Proc. $56^{\text {th }}$ Electronic Components and Technology Conference 2006, p. 1076-1081 (2006).

3. A. Poppe, G. Farkas, Gy. Horváth, Electrical, thermal and optical characterization of power LED assemblies, in Proc. $12^{\text {th }}$ Intern. Workshop on Thermal Investigations of ICs, THERMINIC 2006, p. 197-202 (2006).

4. Yu.N. Davidenko. High-performance modern lightemitting diodes // Sovremennaya Elektronika no 8, p. 36-43 (2004), in Russian.

5. A.E. Belyaev, N.S. Boltovets, R.V. Konakova, Ya.Ya. Kudryk, V.M. Sorokin, V.N. Sheremet, V.V. Shynkarenko, Some aspects of thermal resistance measurement technique for IMPATT and light-emitting diodes // SPQEO 14(4), p. 465-469 (2011).

6. A.V. Zinovchuk, Ya.Ya. Kudryk, Temperature measurement techniques for semiconductor lightemitting diodes // Peterburgskii Zhurnal Elektroniki no 1(66), p. 11-27, 2011, in Russian.

7. A.E. Belyaev, N.S. Boltovets, E.F. Venger, E.G. Volkov, V.P. Klad'ko, R.V. Konakova, Ya.Ya. Kudryk, V.V. Milenin, G.V. Milenin, V.A. Pilipenko, R.A. Red'ko, A.V. Sachenko, Physical Methods of Diagnostics in Micro- and Nanoelectronics, ISMA, Kharkov, 2011, in Russian.

8. A. Keppens, W.R. Ryckaert, G. Deconinck, P. Hanselaer, High power light-emitting diode junction temperature determination from current-voltage characteristics // J. Appl. Phys. 104(9), 093104-093104-8 (2008).

9. O.E. Belyaev, M.S. Boltovets, R.V. Konakova, Ya.Ya. Kudryk, V.M. Sheremet, A method of measurement of thermal resistance of semiconductor diodes // Patent for Useful Model No 65395, Ukraine, 12.12.2011 (in Ukrainian).

10. LUW W5AM Golden DRAGON Plus datasheet. http://catalog.osram-os.com

11. SI Brochure, $8^{\text {th }}$ ed., Appendix 2. The photometric base unit - the candela // Bureau International des Poids et Mesures. 2006. STEDI Media, Paris. http://www.bipm.org/utils/en/pdf/SIApp2_cd_en.pdf 